\section{MACHINE TOOL WITH UNDISTURBED FRAME}

\author{
J. Sveda', Z. Sika' ${ }^{2}$, M. Valasek ${ }^{2}$ \\ ${ }^{\prime}$ Research Center of Manufacturing Technology, \\ CTU in Prague; Czech Republic \\ 2 Department of Mechanics, Biomechanics and Mechatronics, \\ CTU in Prague, Czech Republic \\ e-mail: J.Sveda@rcmt.cvut.cz
}

One of the main disturbances of the machine tool frame is caused by operation of motion axis. Movement of high dynamic motion axis is connected with powerful force strokes that excite machine tool frame vibration and deteriorate machining surface quality and productivity. There are several possibilities how to suppress this vibration. One possibility is to use suspended motor, where the motor is connected with the machine body by spring and damper. This paper deals with new more advanced conception where the main motor is connected with the frame by an active element, that is controlled by intelligent control technique. The active element represents another linear motor, therefore the conception is shortly called "motor on motor".

Keywords

Machine tool, linear motor, vibration suppression, control loop

\section{Introduction}

Contemporary machine tool feed drive construction is based on typical conception that rigidly connects static part of actuator and machine tool body. In case of the linear motor the secondary part is directly connected to the frame. It causes high value and wide frequency spectrum of reaction force that is generated by the control system with high parameter setting. The power strokes are directly transmitted to the machine bed during the operation and wide spectrum of the frequencies are excited. This is connected with amplification of vibration. Oscillation of the machine bed and motion axis then deteriorates precise machining and dynamic properties of motion axis as well as the whole machine tool.

The paper deals with the advanced technique which transforms the frequency spectrum and amplitude of the reaction force. It is based on serial connection of two linear motors in each motion axis with advanced control technique. It allows to suppress the machine tool vibrations and thereby to improve dynamic properties of the motion axis.

\section{Feed drive engineering solution}

Generally there are three principles of feed drive design in machine tool branch including new method described in the paper.

\section{a) Fixed-mounted feed drive}

Fixed-mounted feed drive construction is commonly used in the machine tool branch. As shown in Figure 1, the linear motor is rigidly inbuilt to the machine bed and presents a source of the motion force which reacts equally to the machine frame and motion axis. The primary part is rigidly connected with the motion axis, in this case with the

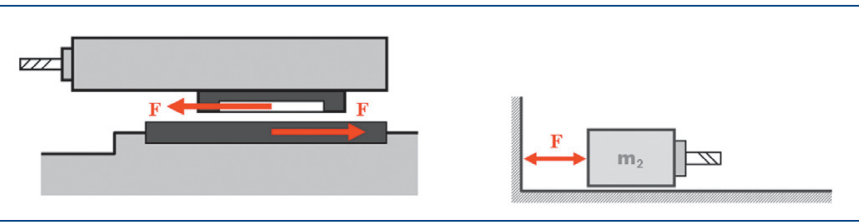

Figure 1. Fixed-mounted feed drive design spindle of the machine tool and the secondary part is fixed-mounted to the machine tool bed.

\section{b) Suspended feed drive}

The suspended feed drive construction [Bubak 2004] is a more sophisticated method. The configuration is shown in Figure 2 and it consists of similar parts as in the previous conception. The primary part of the linear motor is also directly connected with the spindle. However, the secondary part is connected with the machine bed in a different way compared to the previous case. There are spring and damper used for axial fixation to the bed. This configuration allows a short movement of the linear motor secondary part and thus the frequency spectrum of the reaction force is more friendly to the machine bed vibration.

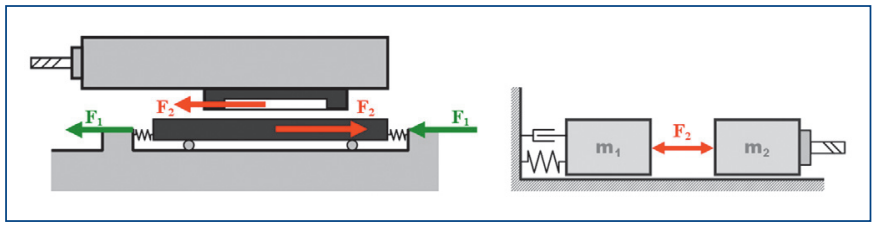

Figure 2. Suspended feed drive design

\section{c) Active feed drive - "motor on motor"}

"Motor on motor" is a new feed drive design. As shown in Figure 3, this concept consists of two independent linear motors. One of them (F2) represents common feed drive which causes displacement of motion axis. The second one deals with the reaction force transformation (F1). The main advantage of this solution is a possibility to fully control the force which reacts to the machine tool body. Thus we can transform the reaction force in an effort to suppress vibrations of the body structure. The paper deals mainly with this concept.

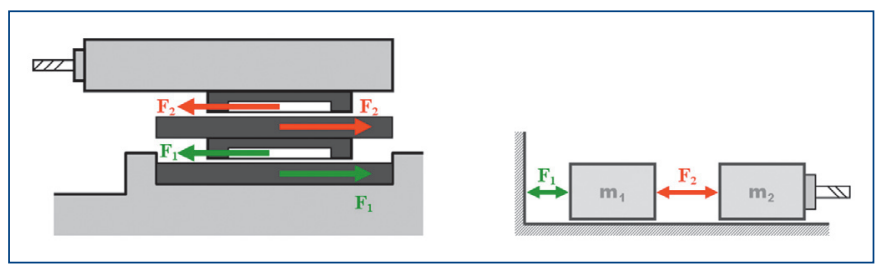

Figure 3. Motor on motor design

\section{Control technique}

\section{a) Impulse decoupling technique}

High efficiency machining is characterized by a low part of other operation time. Therefore the non-manufacturing movement has to be extremely quick and with high acceleration. In such operation the actuator force shape is extremely sharp, ideally it can look like a force step. Unfortunately this impulse frequency spectrum is very wide and many frequencies are excited. It is closely connected with excitation of unfavourable vibrations.

In the new feed drive design the motor (F2) can be controlled by common cascade controller because the force of linear motor is not significantly dependent on the actual velocity of the secondary part of linear motor [Bubak 2004]. The main idea of the control techni-

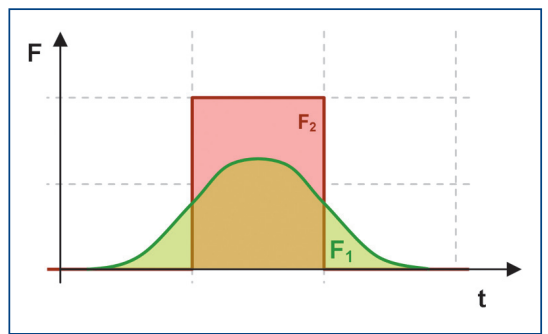

Figure 4. Force time behavior 
que is to prepare the best condition for the axis displacement by means of additional mass movement. It is achieved by the additional force (F1) that operates earlier than the control system demands change of the position. The NC code corresponding to the actuator (F2) has to be known because of the efficient control of the actuator (F1). Thus we can prepare better initial condition for the motion of the main axis and profit from the movement of the additional motor mass (m1).

The time behavior of the mentioned forces can look like in Figure 4. As shown in Figure 4, force which acts on the bed (F1) has smoother time behavior and starts to operate earlier than the force (F2) which reacts to the spindle. This impulse decoupling system decreases force amplitudes which reacts to the bed and reduces number of excited frequencies. We can see that the described impulse decoupling is a technical implementation of an active non-causal filtration of the machine tool bed reaction.

\section{b) Force impulse optimization}

Because the reaction force (F1) is now fully controlled there is used a mathematical method to find its best shape [Valasek 1995], [Stejskal 1996].

The first mathematical method is based on exploitation of genetic algorithm to find an absolute extreme of the target function (1)

$$
\text { tf }=c_{1} \cdot \sum_{i=1}^{n} F_{i}^{2}+c_{2} \cdot \max _{0 \rightarrow i}\left(x_{1}^{2}\right)+c_{3} \cdot \sum_{i=1}^{n-1}\left(F_{i+1}-F_{i}\right)^{2}
$$

The genetic algorithm intelligently computes value of the target function for a large number of force shapes. The results shown in Figure 5 represent target function solution in relation to the reaction force and middle mass displacement. From our point of view the solutions located on the red line are interesting, this solutions are called "pareto-set". Here are both observed criterions minimal, that means efficiency of the method is maximal. Solutions outside the red line are unnecessary and extend computation time.

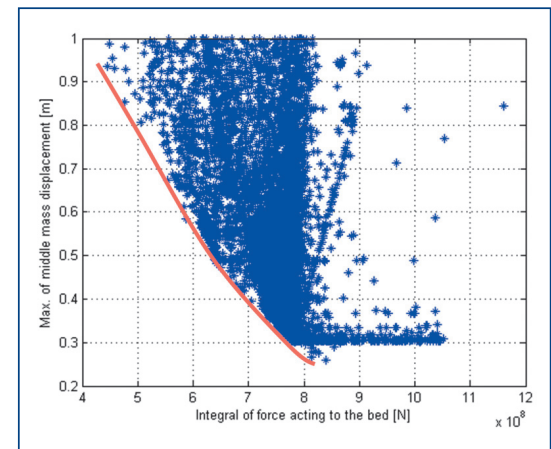

Figure 5. Genetic algorithm optimization results

The second mathematical method exploits quadratic programming algorithm. It solves convex quadratic function using Dantzig-Wolfe's algorithm

$$
\min \frac{1}{2} x^{T} H x+f^{T} x
$$

subject to

$$
A x \leq b, x \geq x_{\min }
$$

The method is also based on the computation of extreme of a target function (4) that has to be in the form of quadratic function written by means of $H$ and $f$.

$$
\text { tf }=c_{1} \cdot \sum_{i=1}^{n} F_{i}^{2}+c_{2} \cdot\left(\sum_{i=1}^{n-1}\left(F_{i+1}-F_{i}\right)^{2}+F_{1}^{2}+F_{n}^{2}\right)+c_{3} \cdot \sum_{i=1}^{n} x_{1 i}^{2}
$$

The greatest advantage of the method is very low time consumption and extremely high efficiency. There are not extended solutions, on- ly the results with minimum criterions are computed. The optimization results are shown in the Figure 6.

Time behavior of the optimized force is shown in the Figure 7. Shown characteristics are relevant to the selected equivalent points from the pareto-sets of the each optimization method. As shown in the Figure 7, the time behavior of the reaction force is similar in both optimization principles. The final shape of the reaction force is also equivalent to the designed shape in Figure 4.

Comparison of the reaction force between the fix, spring and active feed drive design is shown in the Figure 8 . The suspended feed drive transforms the reaction force shape but the force amplitude has very high value and is connected with powerful oscillation.

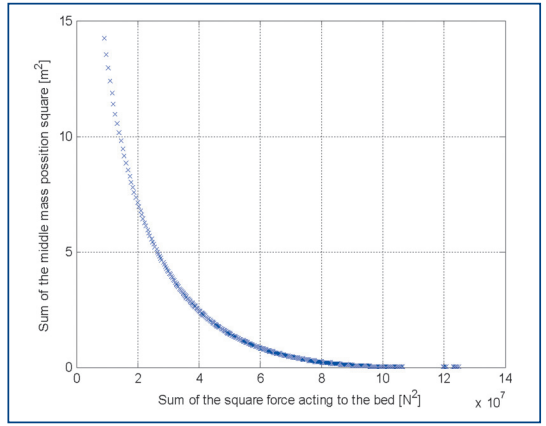

Figure 6. Quadratic algorithm optimization results

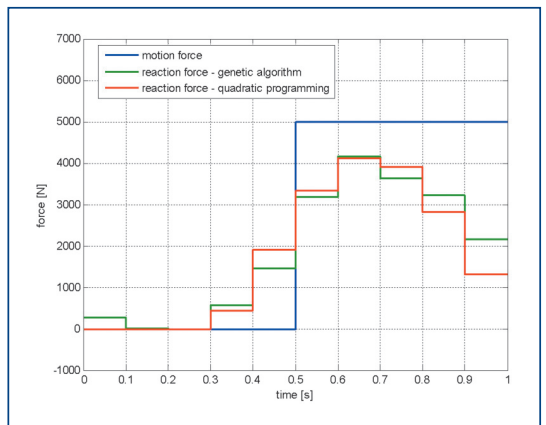

Figure 7. Optimized force time behavior

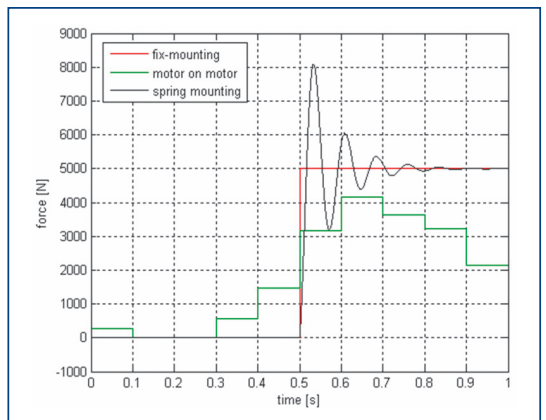

Figure 8. Impulse decoupling comparison

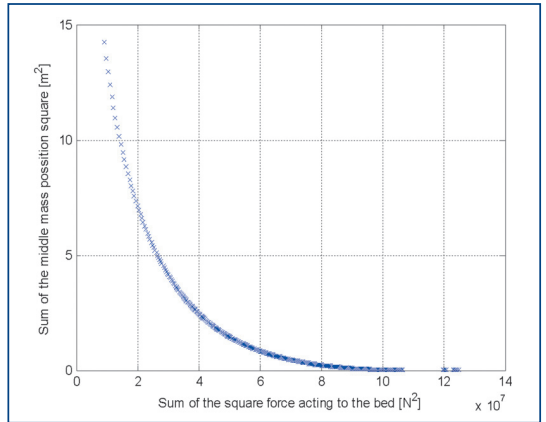

Figure 9. Power spectral density comparison 
There is force discretization for simpler optimization of demanded force shape. The final force shape is interpolated by a cubic spline in each time interval.

The important issue which gives us information about the method quality is the power spectral density of the reaction force. As shown in Figure 9 many frequencies are excited by the application of fixed-mounted principle. It is caused by the sharp profile of the reaction force. Better situation is by the application of the suspended feed drive. The time behavior of the reaction force is not so sharp and lower number of frequencies is excited. Finally the best solution is based on the "motor on motor" conception. As shown in Figure 9 number of excited frequencies is minimal and their amplitudes are lower then by the other conceptions.

\section{c. Regulation scheme}

Regulation scheme is generally splitted into two parts. The first one is common regulation of the motion axis. It can be based on common cascade regulation with position and velocity loop. The second part of the regulation is focused on reaction force transformation through middle mass movement proposed in the previous chapter. It is also divided into two parts where the first one deals with pure non-causal filtration and the second one with absolutization. Both parts cooperates very closely.

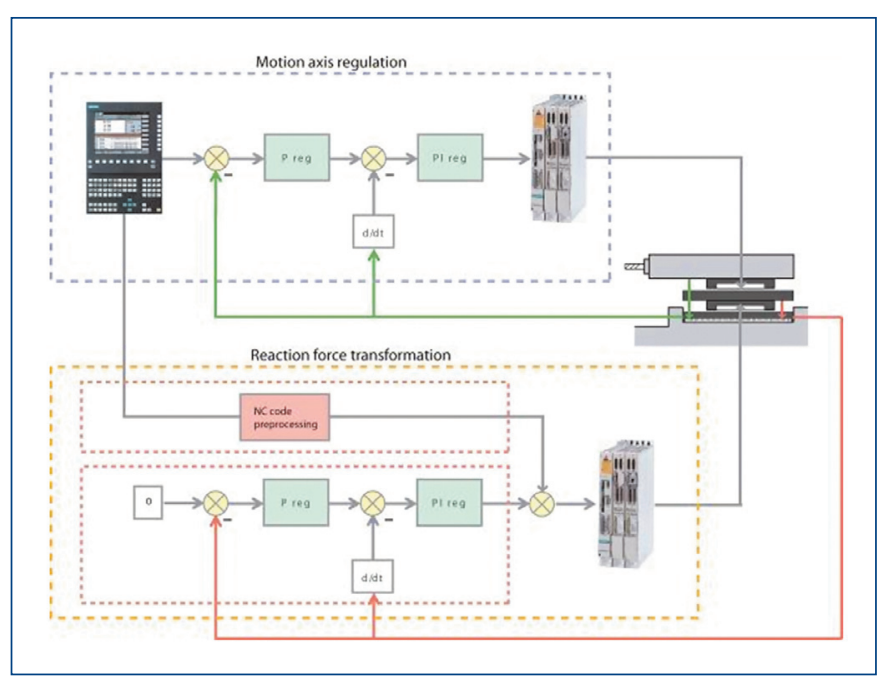

Figure 10. Regulation scheme

The non-causal filtration regulation part is based on NC code preprocessing designed in the previous chapter. Based on the simulation model, the NC code (demanded position) is earliest transformed with the aim of preparing the best initial condition of the feed drive. The absolutization part is then an independent regulation loop with lowered dynamic parameters. Its main purpose is to hold the middle mass position in restricted range in case of disturbance force and mathematical model inaccuracy.

\section{Simulation}

The experimental milling center LM-2 was chosen for simulation testing of the method. This machine has 3-highly dynamical axes equipped with linear motors with possibility to involve suspended feed drive in $\mathrm{Z}$ axis. A complete FEM and simulation model of $\mathrm{LM}-2$ was created, so it is possible to make simulation tests of the method and check its influence on the vibration suppression.

\section{a) Machine tool model}

The machine tool FEM model is created by shell elements and it contains all important parts of the machine tool. In Figure 12 is shown FEM model with highlighted Z-axis which is mathematically equipped with motor on motor conception.

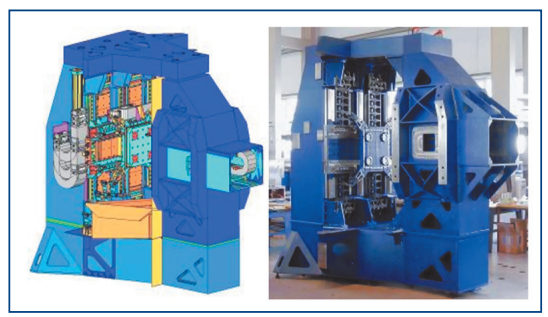

Figure 11. LM-2 machine tool

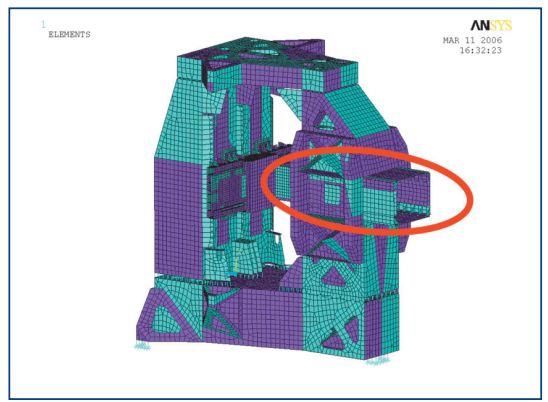

Figure 12. FEM model of LM-2 machine tool

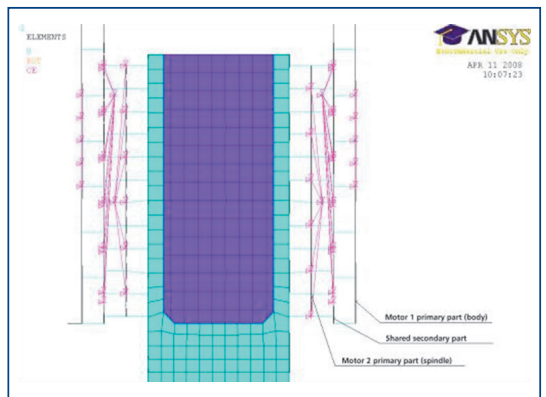

Figure 13. Motor on motor FEM implementation

The detailed motor on motor construction in the FEM model is shown in Figure 13. Movement of the spindle and middle mass (shared secondary part of the linear motors) is allowed by means of extremely low springs stiffness in Z-axis.

Modal analysis of the described FEM model is performed and modal matrix $\Phi$ with eigen frequency vector $\omega$ are stored.

Because we have modal analysis results and the common dynamic equation that solves machine tool dynamic looks like equation

$$
M \ddot{x}_{d}+C \dot{x}_{d}+K x_{d}=f
$$

it is necessary to involve substitution [Milacek 2001]

$$
x_{d}=\Phi_{z}
$$

where $\Phi$ is modal matrix and $z$ is vector of modal coordinate. We obtain modified equation (7) by connection of equations (5) and (6).

$$
M \Phi \ddot{z}+C \Phi \dot{z}+K \Phi z=f
$$

The modal matrix is normed by the mass matrix and assuming symmetry matrices $M$ and $K$ we can write

$$
\Phi^{T} M \Phi=E
$$

$$
\Phi^{T} K \Phi=\operatorname{diag}\left(\omega_{i}^{2}\right)
$$

By multiplying equation (7) with matrix $\Phi^{\top}$ from the left side and using relations (8) a (9) we obtain 


$$
\ddot{z}+\Phi^{T} C \Phi \dot{z}+\operatorname{diag}\left(\omega_{i}^{2}\right) z=\Phi^{T} f
$$

By substitution equation (11)

$$
\Phi^{T} C \Phi=\operatorname{diag}\left(2 \xi_{i} \omega_{i}\right)
$$

in the equation (10) we obtain

$$
\ddot{z}+2 \xi \Omega \dot{z}+\Omega^{2} z=\Phi^{T} f
$$

where

$$
\xi=\operatorname{diag}\left(\xi_{i}\right) \text { and } \Omega=\operatorname{diag}\left(\omega_{i}\right)
$$

Equation (12) describes machine tool dynamic with exploitation of modal matrix and eigen frequency vector. It can be easily transformed into state space approach (14) which is suitable for Matlab/Simulink simulations [5].

$$
\begin{aligned}
& \dot{x}=\left[\begin{array}{c}
\ddot{z} \\
\dot{z}
\end{array}\right]=\left[\begin{array}{cc}
-2 \xi \Omega & -\Omega^{2} \\
1 & 0
\end{array}\right]\left[\begin{array}{l}
\dot{z} \\
z
\end{array}\right]+\left[\begin{array}{c}
\Phi^{T} \\
0
\end{array}\right] f=A x+B f \\
& y=\left[\begin{array}{cc}
\Phi & -0 \\
0 & \Phi
\end{array}\right]\left[\begin{array}{l}
\dot{z} \\
z
\end{array}\right]=C x
\end{aligned}
$$

Described state space model connected with control algorithm designed in chapter 3.3 presents virtual model of machine tool LM-2 with motor on motor conception. Simulation tests were performed on it and the results are described in the following chapter.

\section{b) Simulation results}

Position ramp of the Z-axis with spindle was performed as a first testing set. Before the testing simulation demanded position was transformed into a force impulse by means of non-causal filtration mentioned in chapter 3 . The final shape of the force impulse in comparison with the full reaction force is shown in Figure 14 and the simulated position ramp in Figure 15.

Machine tool body reaction force with an active absolutization control loop is shown in Figure 16. We can see that the new reaction force has extremely low amplitude and smooth shape. Thanks to

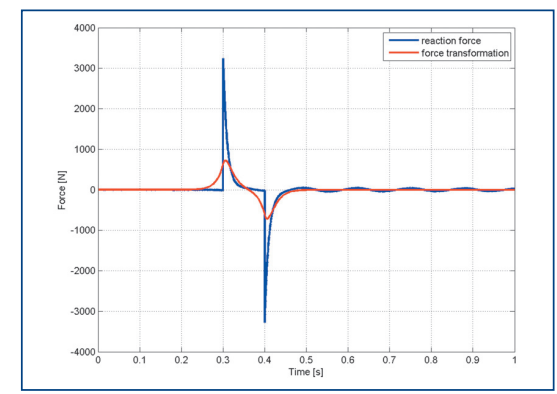

Figure 14. Force impulse and reaction comparison

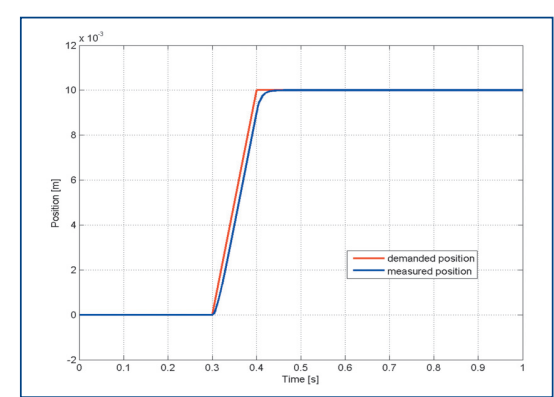

Figure 15. Position ramp

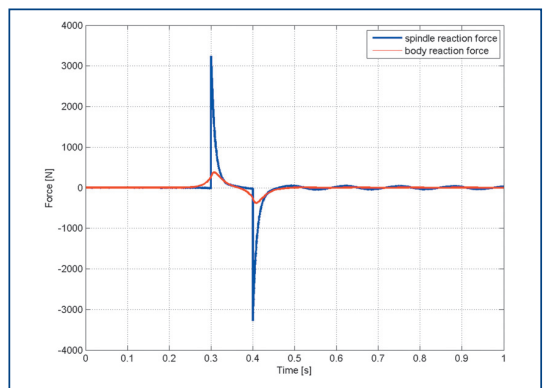

Figure 16. Reaction force with active absolutization

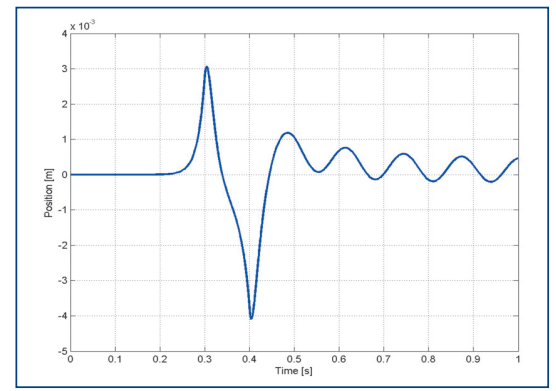

Figure 17. Middle mass displacement

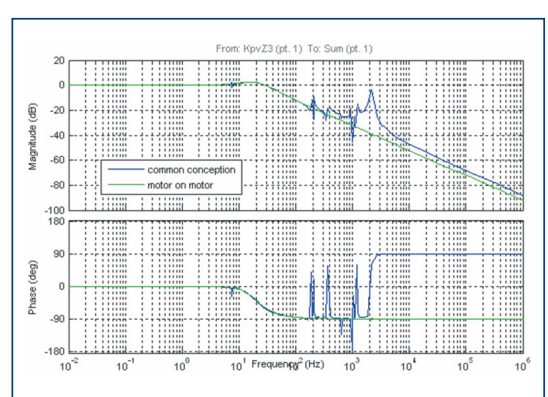

Figure 18. Velocity loop Bode plot

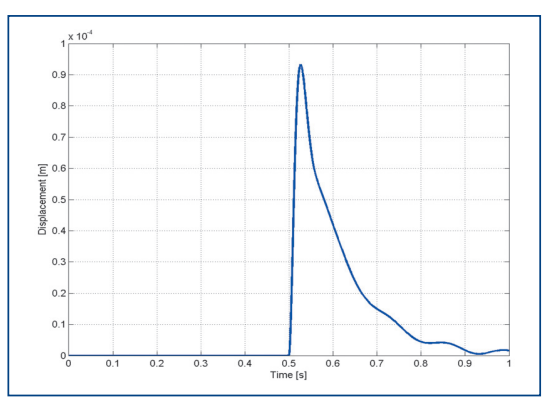

Figure 19. Dynamical stiffness - spindle displacement

it machine tool structural shapes are not excited and position loop gain Kv can be tuned.

Important information about the method quality is also middle mass displacement. This displacement has to be minimal however long enough. The middle mass displacement associated with the position ramp is shown in Figure 17. From the figure flows that we can achieve extremely high dynamical parameters by small control displacement of the middle mass.

The same result is also visible from the velocity loop Bode plot in Figure 18. Motor on motor conception does not excite structural shapes of machine tool by high dynamic movement thus the dynamic parameters can be improved.

The last two figures represents dynamical stiffness test that give us information about disturbance force immunity. In the Figure 19 is shown parasite displacement of the motion axis by force disturbance amplitude $500 \mathrm{~N}$. In the Figure 20 is then shown middle 


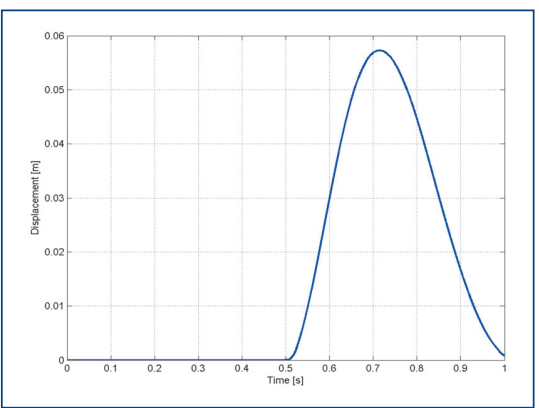

Figure 20. Dynamical stiffness - middle mass displacement

mass movement. The motor on motor conception, that is characterized by series connection of two linear motors, improves motion axis dynamical stiffness by means of position loop gain Kv improvement. Nevertheless it is connected with bigger displacement of the middle mass caused by "lazy" absolutization.

\section{Conclusion}

The designed technology for the impulse decoupling brings new level of the motion dynamic to the machine tool branch. We can achieve reduction of the reaction forces and excited frequencies by exploitation the new decoupling principle. The machine tool equipped with the "motor on motor" conception will be protected from the vibrations and allows high dynamic and precise machining.

The technology is based on the possibility to fully control the reaction force. It is possible thanks to the additional active actuator with relative small stroke that is in series connected to the main motion motor.

The main target of the paper is to introduce the method and its exploitation in machine tool branch. The paper also describes control loop design with absolutization and simulation tests on the experimental milling center LM-2.

Control loop optimization and mathematical model with friction are researched at this time.

\section{Acknowledgement}

The authors appreciate the kind support by MSMT project MSM 6860770003 "Development of algorithms of computational simulations and their applications in engineering".

\section{References}

[Bubak 2004] Bubak, A.: High dynamic and high precision of the NC feed axes. PhD Thesis, Prague, 2004.

[Milacek 2001] Milacek, S., Modal analysis of mechanical vibration. CTU Publishing, Prague, 2001

[Preumont 1997] Preumont, A.: Vibration Control of Aktive Structures. An Introduction, Solid Mechanics and Its Applications, Vol. 50, Kluwer Academic Publisher, Dordrecht, 1997.

[Stejskal 1996] Stejskal, V., Valasek, M.: Kinematics and Dynamics of Machinery. Marcel Dekker, INC., New York.E. Sparrow, Forced convection heat transfer in a duct having spanwise-periodic rectangular protuberances, Numerical Heat, 1996.

[Valasek 1995] Valasek, M., Mechatronics. CTU Publishing, Prague, 1995.

\section{Contacts:}

Jiri Sveda, CTU in Prague

Research Center of Manufacturing Technology

Horska 3, 12800 Praha 2, Czech Republic

tel.: +420 221990 0900, fax: +420221990999

e-mail: J.Sveda@rcmt.cvut.cz 